\title{
Implicações de doenças cardiovasculares na evolução de prognóstico em pacientes com covid-19
}

\author{
Implications of cardiovascular diseases in the evolution of prognosis in patients with \\ covid-19
}

Implicaciones de las enfermedades cardiovasculares en la evolución de pronóstico en pacientes con covid-19

Igor dos Santos Cavalcante ${ }^{1 *}$, Cláudio Vinícius Barroso Queirós de Lima ${ }^{1}$, João Pedro Sousa Mendes $^{1}$, João Victor Carvalho Barbosa', Olívio Joaquim Fonseca Neto ${ }^{1}$, Priscylla Frazão Rodrigues $^{1}$, Yasser da Silveira Krüger ${ }^{1}$, Érica de Araújo Silva Mendes ${ }^{1}$, Elias de Carvalho Magalhães Neto', Renata Paula Lima Beltrão'.

\section{RESUMO}

Objetivo: Identificar e apresentar a relação entre doenças cardiovasculares e os desfechos em pacientes acometidos pela COVID-19. Métodos: Trata-se de um estudo de revisão literária integrativa com coleta de dados realizada nas bases Scientific Eletronic Library Online (SciELO), Literatura Latino-Americana e do Caribe (LILACS) e PubMed. Os dados foram analisados e discutidos diante da literatura atual e pertinente sobre a temática. Resultados: A literatura retrata uma relação direta existente entre comorbidades, especialmente cardiovasculares, e os desfechos da COVID-19. Aspectos fisiopatológicos da infecção por SARS-COV-2 demonstram o papel da Enzima Conversora de Angiotensina - 2 na internalização do vírus pelas células humanas, com destaque para as miocárdicas. Evidenciam-se a relação da COVID-19 com mecanismos de lesão cardíaca e as possíveis manifestações decorrentes disso. Considerações Finais: Pacientes portadores de patologias cardíacas têm maior risco de evolução grave e óbito quando infectados pelo SARS-CoV-2, em comparação ao grupo sem doença cardíacas, possivelmente associados a presença e ação dos receptores enzimáticos conversores de angiotensina. Ademais, alguns pacientes desenvolveram lesões cardíacas secundárias pós-infecção.

Palavras-chave: Infecções por coronavírus, Doenças cardiovasculares, Fatores de risco.

\section{ABSTRACT}

Objective: To identify and to present the correlation between cardiovascular diseases and the outcomes in patients affected by COVID-19. Methods: This is an integrative literary review study in which data collection was carried out in the Scientific Eletronic Library Online (SciELO), Latin American and Caribbean Literature (LILACS) and PubMed databases. The data were analyzed and discussed with the current literature. Results: The literature shows an existing direct correlation between comorbidities, especially cardiovascular diseases, and the outcomes of COVID-19. SARS-CoV-2 infection pathophysiology and its characteristics evidence the role played by angiotensin II converting enzyme (ACE II) on the virus internalization by host cells, mainly cardiac cells. In this regard, data and researches point to the correlation between COVID-19 and cardiac injury mechanisms so as the possible manifestations as a result of that. Final Considerations: Patients with cardiac pathologies are more likely to die when infected by SARS-CoV-2, of which about half died and it is estimated to be due to angiotensin-converting enzyme receptors. In addition, some patients developed secondary cardiac injuries after infection.

Keywords: Coronavirus infections, Cardiovascular diseases, Risk factors.

1 Universidade Federal do Delta do Parnaíba (UFDPAR), Parnaíba - PI. *E-mail: igorsc@live.com 


\section{RESUMEN}

Objetivo: Identificar y presentar la relación entre las enfermedades cardiovasculares y los resultados en pacientes afectados por COVID-19. Métodos: Este es un estudio integrador de revisión literaria en el que la recopilación de datos se llevó a cabo en las bases de datos de la Biblioteca Científica Eletrónica en línea (SciELO), Literatura Latinoamericana y del Caribe (LILACS) y PubMed. Los datos fueron analizados y discutidos con la literatura actual. Resultados: La literatura retrata una relación directa entre las comorbilidades, especialmente las cardiovasculares, y los resultados de COVID-19. Los aspectos fisiopatológicos de la infección por SARS-CoV-2 muestran el papel de la enzima convertidora de angiotensina 2 en la internalización del virus por las células humanas, especialmente las células cardíacas. En este sentido, son evidentes los datos y la investigación que vinculan COVID-19 con los mecanismos de lesión cardíaca y las posibles manifestaciones resultantes de esto. Consideraciones Finales: Los pacientes con patologías cardíacas son más propensos a la muerte cuando se infectan con SARS-CoV-2, de los cuales aproximadamente la mitad murió y se estima que se deben a receptores de enzimas conversoras de angiotensina. Además, algunos pacientes desarrollaron lesiones cardíacas secundarias después de la infección.

Palabras clave: Infecciones por coronavirus, Enfermidades cardiovasculares, Factores de riesgo.

\section{INTRODUÇÃO}

Em dezembro de 2019, em Wuhan, na China, o crescente número de indivíduos apresentando complicações respiratórias de causa desconhecida alertou profissionais e autoridades públicas. Estudos apontaram que a origem da disseminação seria o mercado de frutos do mar, e que se tratava de um novo agente da família Coronaviridae, denominado posteriormente de Coronavírus da Síndrome Respiratória Aguda Grave 2, com a sigla SARS-CoV-2 (ZHOU F, et al., 2020; ZHENG Y-Y, et al., 2020). Logo, a transmissão do vírus foi atribuída a gotículas respiratórias de pessoas contaminadas, sintomáticas ou não, com a possibilidade menos provável de aerossóis e contato com as fezes (CLERKIN KJ, et al., 2020).

A elevada capacidade de transmissão, mesmo com virulência relativamente baixa da SARS-CoV-2, foi responsável pela disseminação mundial e o alcance do status de pandemia, levando à morte milhares de pessoas. (WANG D, et al., 2020; BANSAL M, 2020).

A doença reconhecida pela Organização Mundial da Saúde (OMS) como COVID-19 (do inglês Coronavirus Disease), assimila-se à SARS, de 2002, e à Síndrome Respiratória do Médio Oriente (MERS, do inglês), de 2012 (CLERKIN KJ, et al., 2020).

Atualmente, os impactos nos sistemas de saúde do mundo inteiro, em conjunto com os desafios econômicos e culturais ocasionados por esse vírus, tornaram esta pandemia o maior desafio já enfrentado pela saúde moderna (CRIVELARI NC, et al., 2020; GRANEMANN S, 2021).

A COVID-19 possui espectro clínico heterogêneo, podendo variar entre os quadros: assintomático, leve, moderado ou grave, exigindo cuidados intensivos e suporte avançado respiratório. Entre os principais sintomas estão: tosse seca, febre, anosmia, ageusia, odinofagia e dispneia (WANG D, et al., 2020). Apesar do sistema respiratório ser o mais afetado, o sistema cardiovascular também pode ser atingido, levando a diversas consequências sistêmicas de modo variável (BANSAL M, 2020).

Estudos publicados mostram que as pessoas com algum tipo de doença cardiovascular, como hipertensão arterial e doença arterial coronariana, apresentam prognóstico mais grave quanto a COVID-19 e um maior risco de morte, o que acentua a necessidade de manejo adequado (ZHENG Y-Y, et al., 2020; COSTA IBS da S, et al., 2020).

Esse fato está relacionado ao mecanismo de internalização do vírus em células humanas, devido à atuação da enzima conversora de angiotensina 2 , a qual funciona como um receptor da proteína estrutural $S$ (espícula) do vírus, o que irá ocasionar um conjunto de alterações na conformação celular, demonstrando como a presença de patologias cardiovasculares pode desencadear um desfecho potencialmente negativo para os pacientes (CHENG P, et al., 2020). 
Dentre os fatores de risco para a doença destacam-se: idade acima de 60 anos, como principal fator, seguida de pessoas portadoras de doenças crônicas, como diabetes e hipertensão arterial, e, ainda, pacientes com imunidade suprimida, a exemplo dos pacientes oncológicos (KAHARA LT, et al, 2020).

A população idosa possui maiores taxas de mortalidade associada, porque além do fator de envelhecimento inerente, ainda se associa esse grupo a uma elevada prevalência de doenças crônicas (BORGES GM e CRESPO CD, 2020).

Em pesquisa inédita realizada por Barrasa $\mathrm{H}$, et al. (2020), as comorbidades mais comuns apresentadas pelos pacientes foram obesidade (48\%), hipertensão arterial (44\%) e doença pulmonar crônica (37\%). Ainda, em estudo feito por Piva S, et al. (2020), observou-se a presença de hipertensão em $45 \%$ dos indivíduos analisados com COVID-19 nas Unidades de Terapia Intensiva. Esses dados corroboram com os indícios de que há uma maior susceptibilidade para complicações em pacientes com doenças cardiovasculares.

Dessa forma, o presente artigo busca identificar e apresentar a relação entre doenças cardiovasculares e seus pacientes acometidos pela COVID-19.

\section{MÉTODOS}

Trata-se de uma revisão de literatura integrativa, obedecendo a normas pertinentes de levantamento, de seleção, de ordenação e de síntese de material publicado por outros autores. Para este fim foi determinada a seleção do tema e definição do objetivo de pesquisa. Posteriormente, foram determinados os fatores de inclusão bem como os de exclusão.

Dessa forma, o tema definido foi "doenças cardiovasculares e COVID-19" e objetivou-se apresentar os impactos que as doenças cardiovasculares exercem na evolução de prognóstico negativo em pacientes portadores do Covid-19, visando apresentar e analisar quais particularidades essas patologias possuem no agravante da infecção pelo vírus.

Foi realizada uma pesquisa bibliográfica nas bases de dados Scientific Eletronic Library Online (SciELO), Medical Literature Analysis and Retrievel System Online (Medline) e Literatura Latino-Americana e do Caribe (LILACS), utilizando os descritores: "Infecções por coronavírus (COVID-19)", "Doenças cardiovasculares" e "Fatores de risco". Para aprimoramento da busca foi utilizado o operador booleano "AND", e selecionados artigos de relevância para o tema.

Ao utilizar os três descritores associados na base Lilacs, nenhum artigo foi encontrado e, ao utilizar o descritor "infecções por coronavírus" associado, pelo fator booleano, ao descritor "fatores de risco", obtevese quatro (4) artigos, já a combinação "fatores de risco" e "doenças cardiovasculares" não esteve presente entre as pesquisas para evitar o tangenciamento do tema em questão.

Na base Medline, a combinação dos três descritores resultou em sete (7) artigos, utilizando-se associação "infecções por coronavírus" e "doenças cardiovasculares" foram encontrados trinta e um (31) artigos, com o par "infecções por coronavírus" e "fatores de risco" foi possível obter cento e noventa (190) artigos. Da mesma maneira, o par "doenças cardiovasculares" e "fatores de risco" foi descartado para não haver divergência com o tema relacionado ao coronavírus.

$\mathrm{Na}$ base SciELO, a pesquisa dos três descritos juntos, ofereceu três (3) artigos. Um (1) artigo foi encontrado ao pesquisar o par "infecções por coronavírus" e "doenças cardiovasculares", enquanto que com a combinação "infecções por coronavírus" e "fatores de risco", foi possível encontrar quatro (4) artigos. Seguindo o padrão das demais bases de dados, o par "doenças cardiovasculares" e "fatores de risco" foi descartado, evitando-se a divergência com o tema abordado na pesquisa.

Os critérios de inclusão foram: artigos científicos disponibilizados de forma gratuita e integral, no inglês ou português do Brasil, que fossem pesquisas em seres humanos e publicados no ano de 2020, cujo tema abordado tratasse de problemas cardíacos relacionados à COVID-19. 
Quanto aos critérios de exclusão foram desconsiderados materiais que não fossem artigos científicos, não estivem dentro do período de 2020 e estudos que não se relacionavam ao tema selecionado (Figura 1).

Figura 1 - Fluxograma detalhando cada etapa do processo de seleção de artigos para a revisão.
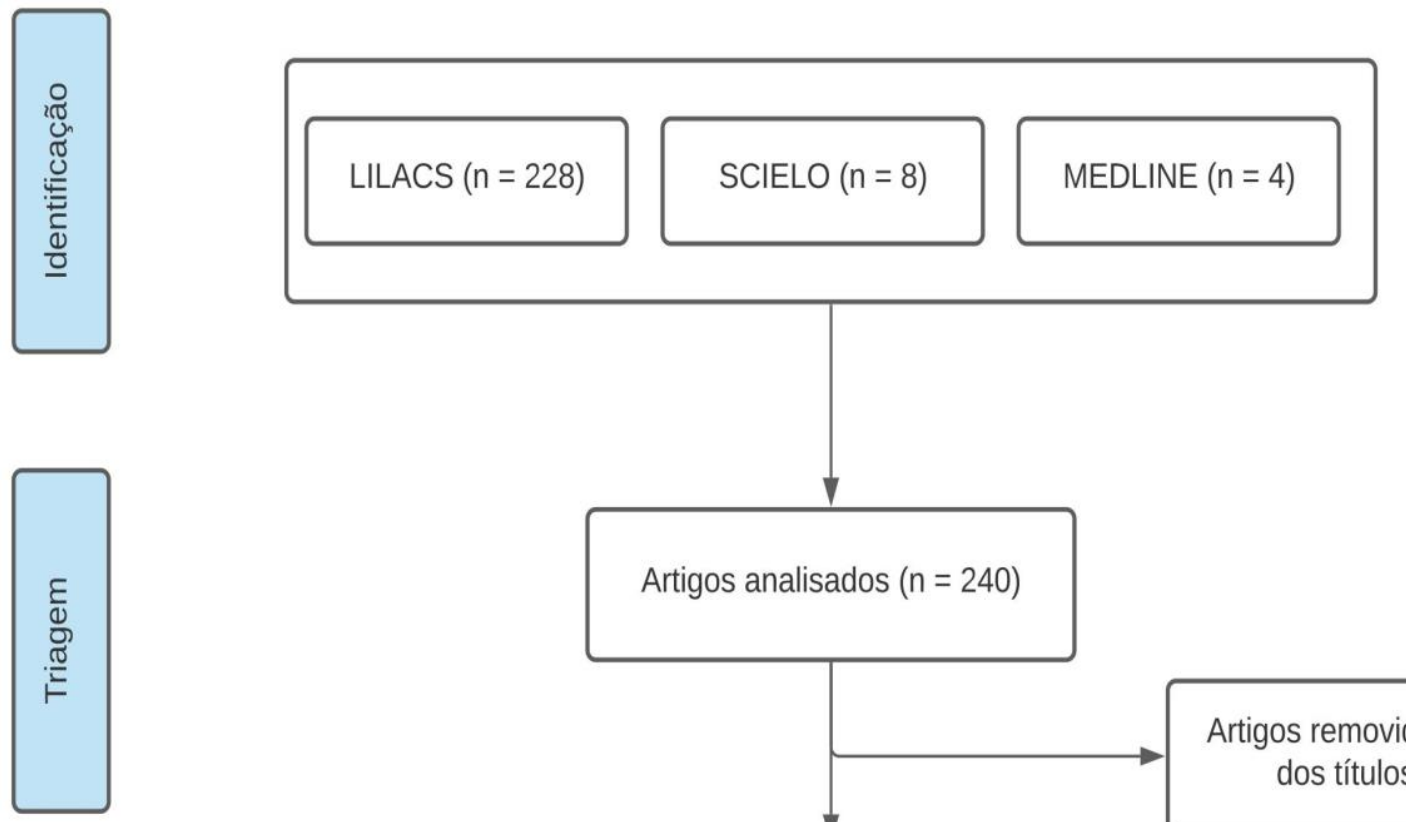

Artigos analisados $(n=240)$
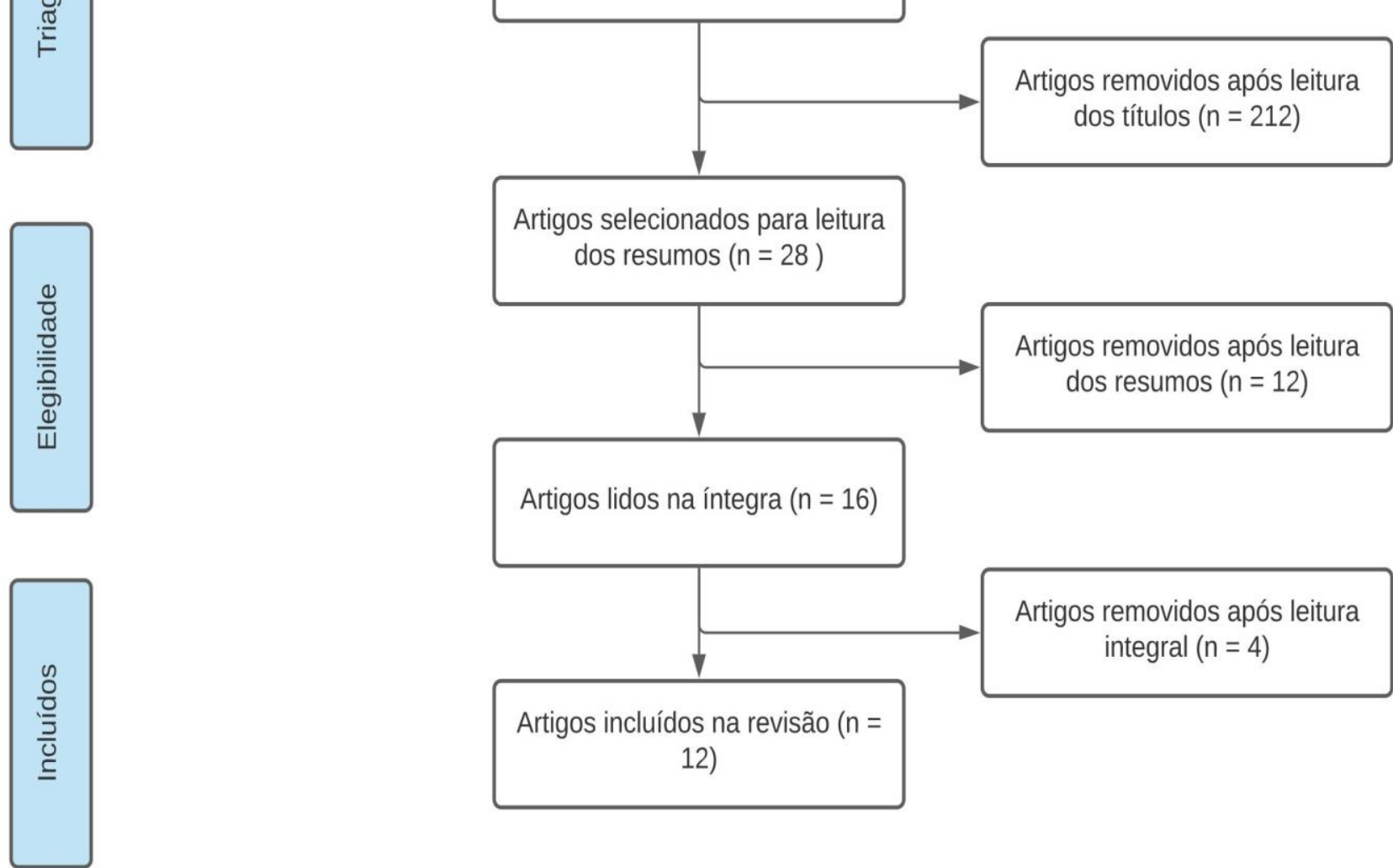

Artigos lidos na íntegra $(n=16)$

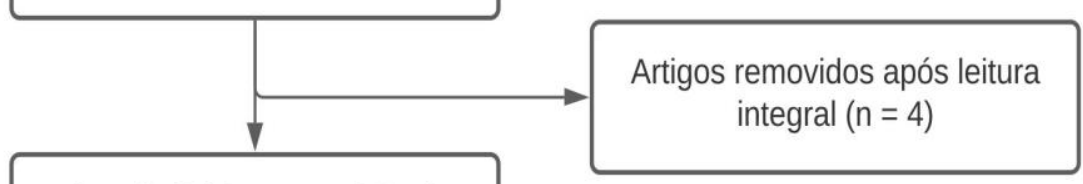

Artigos incluídos na revisão $(\mathrm{n}=$ 12)

Fonte: Cavalcante, IS, et al., 2020.

\section{RESULTADOS}

A seleção dos estudos foi realizada de forma independente por dois pesquisadores, a partir da análise dos títulos, resumos e textos completos das publicações. Encontrou-se ao todo 240 artigos na busca primária. Inicialmente, duzentos e dezesseis (212) artigos, cujos títulos fugiam do tema, foram descartados, bem como catorze (14) que, após leitura do resumo, não se enquadraram nos fatores de inclusão. A seguir, com a leitura completa dos artigos, foram excluídos dois (2) artigos. Assim, restaram doze (12) artigos para compor a pesquisa final (Quadro 1). 


\section{Revista Eletrônica Acervo Saúde / Electronic Journal Collection Health | ISSN 2178-2091}

Quadro 1 - Artigos selecionados para a composição do estudo, abordando o periódico, ano de publicação e seus respectivos objetivos.

\begin{tabular}{|c|c|c|c|}
\hline Obra & Ano & Periódico & Objetivo \\
\hline $\begin{array}{l}\text { COVID-19, ACE2, and the cardiovascular } \\
\text { consequences }\end{array}$ & 2020 & $\begin{array}{c}\text { The American heart Jounal of } \\
\text { Physiology: Heart and Circulatory } \\
\text { Physiology }\end{array}$ & $\begin{array}{l}\text { Avaliar a relação entre o Covid-19, a proteína ACE2 e as } \\
\text { consequências cardiovasculares. }\end{array}$ \\
\hline $\begin{array}{l}\text { Clinical characteristics of } 113 \text { deceased patients } \\
\text { with coronavirus disease 2019: retrospective study }\end{array}$ & 2020 & British Medical Journal & $\begin{array}{l}\text { Delinear as características clínicas dos pacientes } \\
\text { Com covid-19 que falaceram. }\end{array}$ \\
\hline $\begin{array}{l}\text { Cardiovascular Risks in Patients with COVID-19: } \\
\text { Potential Mechanisms and Areas of Uncertainty }\end{array}$ & 2020 & Current Cardiology Reports & $\begin{array}{l}\text { Revisar a literatura científica acerca dos riscos } \\
\text { cardiovasculares para o covi-19 e seus potenciais } \\
\text { mecanismos. }\end{array}$ \\
\hline $\begin{array}{l}\text { Perspective: cardiovascular disease and the } \\
\text { Covid-19 pandemic }\end{array}$ & 2020 & Basic Research in Cardiology & Relacionar doenças cardiovasculares e o covid-19. \\
\hline $\begin{array}{c}\text { The cardiovascular burden of coronavirus disease } \\
2019 \text { (COVID-19) with a focus on congenital heart } \\
\text { disease }\end{array}$ & 2020 & International Journal of Cardiology & $\begin{array}{l}\text { Averiguar a carga cardiovascular no covid-19, com foco na } \\
\text { doença cardíaca congênita. }\end{array}$ \\
\hline $\begin{array}{l}\text { Clinical course and risk factors for mortality of adult } \\
\text { in patients with COVID-19 in Wuhan, China: a } \\
\text { retrospective cohort study }\end{array}$ & 2020 & The Lancet & $\begin{array}{l}\text { Avaliar o curso clínico e fatores de risco para mortalidade de } \\
\text { adultos internados com COVID-19 em Wuhan, China: um } \\
\text { estudo coorte retrospectivo. }\end{array}$ \\
\hline
\end{tabular}




\section{Revista Eletrônica Acervo Saúde / Electronic Journal Collection Health | ISSN 2178-2091}

\begin{tabular}{|c|c|c|c|}
\hline Obra & Ano & Periódico & Objetivo \\
\hline $\begin{array}{c}\text { Clinical course and outcome of } 107 \\
\text { Patients infected with the novel } \\
\text { coronavirus, SARS-CoV-2, discharged from two } \\
\text { hospitals in Wuhan, China }\end{array}$ & 2020 & Critical Care & $\begin{array}{l}\text { Analisar curso clínico e resultado de } 107 \text { pacientes } \\
\text { infectados com o romance coronavírus, SARS-CoV-2, } \\
\text { descarregado de dois hospitais em Wuhan, China. }\end{array}$ \\
\hline COVID-19 and the cardiovascular system & 2020 & Nature Reviews Cardiology & $\begin{array}{l}\text { Demonstrar a relação entre o sistema cardiovascular e o } \\
\text { covid-19. }\end{array}$ \\
\hline $\begin{array}{c}\text { Coronavírus e o Coração: Um Relato de Caso } \\
\text { sobre a Evolução da COVID-19 Associado à } \\
\text { Evolução Cardiológica }\end{array}$ & 2020 & Sociedade Brasileira de Cardiologia & $\begin{array}{l}\text { Relato de caso que demonstra complicações secundárias à } \\
\text { infecção por COVID-19 devido à acometimento cardíaco. }\end{array}$ \\
\hline Covid-19 e o coração & 2020 & Sociedade Brasileira de Cardiologia & $\begin{array}{l}\text { Relatar dados numéricos em relação aos fatores de risco } \\
\text { associado à COVID-19, além de ressaltar os problemas } \\
\text { cardiovasculares apresentados pelos pacientes. }\end{array}$ \\
\hline $\begin{array}{l}\text { O Coração e a COVID-19: O que o Cardiologista } \\
\text { Precisa Saber }\end{array}$ & 2020 & Sociedade Brasileira de Cardiologia & $\begin{array}{l}\text { Relata informações de como atua o COVID-19 no organismo } \\
\text { com doenças cardiovasculares no intuito de disseminar } \\
\text { informação aos cardiologista para o melhor manejo desses } \\
\text { pacientes. }\end{array}$ \\
\hline $\begin{array}{c}\text { COVID-19: Dados Atualizados e sua Relação Com } \\
\text { o Sistema Cardiovascular }\end{array}$ & 2020 & Sociedade Brasileira de Cardiologia & $\begin{array}{l}\text { Mostrar dados da relação do COVID-19 com doenças } \\
\text { cardiovasculares em diferentes faixas etárias. }\end{array}$ \\
\hline
\end{tabular}

Fonte: Cavalcante IS, et al, 2020. 


\section{Covid-19: quadro clínico e relação com comorbidades}

SARS-CoV-2, betacoronavírus causador da SARS, a princípio, foi especificado e tratado como pneumonia devido à insuficiência ou mesmo à ausência de dados no que tange a sua classificação. Desde sua então recém descoberta e categorização, estudos vêm sendo realizados ao redor do mundo a fim de mitigar os efeitos do vírus em pacientes infectados e de contribuir para sanar o cenário preocupante instalado nos últimos meses.

A COVID-19, doença causada pela infecção por SARS-CoV-2, possui dois estágios clínicos. O primeiro é caracterizado pela replicação viral persiste por alguns dias e pela manifestação de sintomas leves pelos pacientes. Infectados sintomáticos geralmente apresentam febre, tosse seca e dispneia, podendo ter achados de imagem semelhantes à pneumonia na radiografia de tórax. Cerca de 18\% dos pacientes podem, também, ser assintomáticos. O segundo estágio é caracterizado por adaptação imunológica, com o organismo humano desenvolvendo resposta de anticorpos específicos contra o patógeno invasor. Observa-se, nesse estágio, uma diminuição da carga viral e dos sintomas apresentados no primeiro estágio. Todavia, aproximadamente $10 \%$ dos pacientes podem apresentar agravamento de seu estado clínico, exigindo terapia intensiva e cuidados continuados (TAN W e ABOULHOSN J, 2020).

Segundo a Sociedade Brasileira de Cardiologia (SBC), o quadro clínico da doença se assemelha ao de outras viroses que afetam o sistema respiratório, estando, entre os principais sintomas, febre, tosse geralmente seca e cansaço. Casos mais graves, em torno de $5 \%$ do total, podem evidenciar, também, sintomas como sangramento pulmonar, linfopenia grave e insuficiência renal. O diagnóstico de sintomáticos pode ser confirmado, em laboratório, por meio do exame reação em cadeia da polimerase (PCR) a partir da coleta de amostras do swab nasal (STRABELLI TMV e UIP DE, 2020).

Ainda a respeito do quadro clínico, Chen $\mathrm{T}$, et al. (2020) aborda e analisa a prevalência dos sintomas mais comuns da doença, como tosse e febre, presentes em ambos os grupos explorados em seu estudo, mortos e recuperados. Fadiga, expectoração, dispneia e dor torácica estão entre sintomas predominantes, sendo, estes dois últimos, observados, sobretudo, em pacientes que vieram a óbito (CHEN T, et al., 2020). Essa gama de sintomas esteve presente na maioria dos estudos analisados, apresentando-se frequentemente em pacientes infectados pelo SARS-CoV-2.

Com ascensão do novo coronavírus, tornou-se uma preocupação dos médicos a conduta e o manejo corretos dos pacientes os quais apresentam comorbidades, visto que contribuem como fator de risco à nova infecção. A justificativa para isso está na resposta inflamatória incitada pela infecção por SARS-CoV-2, que eleva os níveis séricos de substâncias, como: troponina, peptídeo natriurético cerebral (NT-proBNP), dímeroD, interleucina 6 (IL-6), proteína C-reativa, procalcitonina, entre outras. Consequentemente tem-se a liberação tanto de substâncias pró-inflamatórias como de substâncias marcadoras de lesão cardíaca, provocando reações locais e sistêmicas. Seus efeitos podem resultar em complicações cardiovasculares, em choque e até em óbito (COSTA IBS da S, et al., 2020).

Ainda há a necessidade de melhor esclarecimento acerca das consequências da COVID-19 em pessoas com história de doenças cardiovasculares mesmo já havendo grande mobilização dos pesquisadores. Esses efeitos, em especial em pacientes portadores de cardiopatia congênita, vêm sendo elucidados conforme a investigação sobre a doença avança e conforme há o surgimento de novos estudos da comunidade científica mundial (TAN W e ABOULHOSN J, 2020).

Os estudos supracitados mais atuais retratam que doenças pré-existentes, principalmente as cardiovasculares, relacionam-se diretamente com o quadro clínico e com as manifestações subsequentes ao estabelecimento do vírus no organismo. Estão entre essas comorbidades: hipertensão arterial sistêmica (HAS), diabetes, insuficiência cardíaca (IC), além de outras doenças cardiovasculares, como a doença arterial coronariana (DAC) e a doença cerebrovascular - além de tais enfermidades, a idade avançada também se apresenta como importante fator de risco. Como instrumento de confirmação da influência de doenças préexistentes na reação do paciente à infecção, analisa-se o fato de enfermidades como a hipertensão, a doença cardiovascular e a doença cerebrovascular serem mais comumente atestadas nos indivíduos que faleceram do que no grupo de indivíduos que se recuperaram, tendo em vista que essas pessoas estão mais suscetíveis a desenvolver as formas graves da doença (CHEN T, et al., 2020). 
O estudo retrospectivo de Chen T, et al. (2020) explicita a relação supracitada. Tendo acompanhado 799 pacientes, dos quais 113 doentes faleceram com a infecção por SARS-CoV-2, evidenciou que 48\% apresentavam hipertensão, 14\% doenças cardiovasculares e 4\% doença cerebrovascular.

Já entre os 161 pacientes que, no intervalo de tempo do estudo, receberam alta hospitalar, as porcentagens são, respectivamente, 24\%, 4\% e 0\% (CHEN T, et al., 2020). Ademais, no estudo de Zhou F, et al. (2020), dentre os pacientes que faleceram devido à infecção pelo novo coronavírus, $67 \%$ apresentavam algum tipo de comorbidade. Já entre os sobreviventes, essa taxa é de cerca de $40 \%$.

Diante do exposto sobre o quadro clínico e considerando as comorbidades relacionadas à infecção por SARS-CoV-2, observa-se que há uma forte relação da COVID-19 com o sistema cardiovascular, sendo, essa questão, de importante discussão e detalhamento.

\section{Covid -19 e o sistema cardiovascular}

No escrito seguinte, são abordados os aspectos fisiopatológicos da COVID-19, relacionando a doença a manifestações cardiovasculares e tratando de como o processo de lesão das células cardíacas iniciado pela infecção pode ser identificado por meio de exames laboratoriais.

\section{Sobre a Fisiopatologia}

O novo coronavírus SARS-CoV-2 pode causar significativo prejuízo em pacientes com doenças cardiovasculares. O mecanismo de infecção do SARS-CoV-2 retrata uma forte relação da COVID-19 com a enzima conversora da angiotensina 2 (ECA2), uma vez que o vírus necessita dos receptores dessa enzima para ser internalizado na célula hospedeira, devido a interação na superfície celular com as proteínas $S$ presente no vírus. (FERRARI F, 2020).

Sabendo disso, bloquear ou reduzir a expressão da enzima poderia ser uma nova medida terapêutica no que diz respeito ao intuito de prevenir a infecção em tecidos e em órgãos que expressam a ECA2, como os pulmões, o coração, os rins, o cérebro e o intestino. Particularmente, há uma expressão considerável de ECA2 no coração se comparado aos pulmões, o que pode justificar a incidência alta de lesões cardíacas encontradas em pacientes com a COVID-19 (CHENG P, et al., 2020).

A ECA2, numa série de eventos catalíticos, degrada a angiotensina II (ANGII), que é a maior promotora do sistema renina-angiotensina-aldosterona (SRAA) - sistema que reduz a sensibilidade de barorreceptores através do controle da frequência cardíaca $(F C)$ e da promoção da vasoconstrição, da retenção de sódio, do estresse oxidativo, da inflamação e da fibrose, resultando em hipertensão (SOUTH AM, et al., 2020).

Muitos pacientes idosos com hipertensão ou com outras doenças cardiovasculares são rotineiramente tratados com bloqueadores do SRAA. Isso tem gerado novas preocupações clínicas para que sejam elucidadas lacunas existentes no conhecimento, como: a) se esses pacientes possuem maior risco para a infecção por SARS-CoV-2; b) se o uso de métodos terapêuticos que envolvem o SRAA deve ser interrompido; e c) quais as potenciais consequências do bloqueio do SRAA para patologias relacionadas à COVID-19, tais como doenças respiratórias crônicas e agudas (SOUTH AM, et al., 2020).

Em se tratando de manifestações cardiovasculares, como a lesão cardíaca aguda, o choque e a arritmia, sabe-se que elas podem estar presentes em pacientes infectados pelo vírus, sendo, esses sintomas, os mais prevalentes entre pacientes que necessitaram de cuidados intensivos (FERRARI F, 2020). O mecanismo da lesão cardíaca ainda não está totalmente descrito, mas há dados que documentam os efeitos da COVID-19 no sistema cardiovascular, em especial no acometimento do miocárdio (TAN W e ABOULHOSN J, 2020). Devido à fragilidade e à suscetibilidade a esse acometimento, pacientes portadores de doenças cardiovasculares possuem um risco particularmente elevado de mortalidade por COVID-19 (GORI T, et al., 2020).

Casos que resultaram num quadro de insuficiência cardíaca aguda, por exemplo, constituem uma importante complicação secundária. Não havia, até meados de junho de 2020, tratamento específico para situações como essa a não ser o seguimento das diretrizes já existentes para o tratamento de insuficiência cardíaca aguda somado ao controle da infecção (RENTE A, et al., 2020). 
Já em casos de pacientes com síndrome coronariana aguda que se infectam com SARS-CoV-2, o prognóstico tende a ser desfavorável, podendo haver uma redução da função cardíaca devido à isquemia do miocárdio e à necrose (ZHENG Y-Y, et al., 2020).

Efeitos do Sars-CoV-2 na função microvascular, como lesão pericítica - lesão dos pericitos - têm sido reportados, em particular nos pacientes com insuficiência cardíaca (GORI T, et al., 2020). Além disso, drogas que têm sido utilizadas empiricamente no tratamento da doença, como a cloroquina, possuem efeitos na repolarização do miócito. Elas ocasionam, assim, maior risco de prolongamento do intervalo QT e de arritmia subsequente. De modo geral, lesões no miocárdio e insuficiência cardíaca relacionadas à infecção por SARSCoV-2 constituem particularidades da COVID-19 (CHENG P, et al., 2020).

Torna-se crucial, então, num contexto de alta incidência de anormalidades eletrolíticas em doentes, que o uso de substâncias farmacológicas seja devidamente observado de modo a evitar possíveis danos iatrogênicos. Lesões secundárias decorrentes da infecção por SARS-CoV-2, como a lesão miocárdica ou o desenvolvimento e piora de quadro de insuficiência cardíaca, podem ser rastreadas por meio de exames laboratoriais. Biomarcadores inflamatórios como troponina e NT pro-BNP são exemplos de indicadores dessa disfunção. (CHENG P, et al., 2020).

Como afirma Zhou F, et al. (2020), pacientes com elevados níveis desses marcadores apresentaram uma maior incidência de arritmias ventriculares e a necessidade de ventilação mecânica. Além disso, atesta, em sua pesquisa, que os níveis séricos de troponina I hipersensitiva estavam evidentemente mais elevados nos pacientes que foram a óbito em relação aos pacientes recuperados. Esses níveis, conforme o apresentado, crescem rapidamente com a progressão da doença quando associada ao aumento da gravidade do quadro e à lesão cardíaca. O dano miocárdico é definido se os níveis séricos de troponina estiverem acima do $99^{\circ}$ percentil do valor de referência, $28 \mathrm{pg} / \mathrm{mL}$. Neste coorte, a troponina frequentemente foi observada em pacientes com quadros severos de COVID-19, estando presente, com níveis aumentados, em mais da metade dos pacientes falecidos, atingindo um valor mediano de $22.2 \mathrm{pg} / \mathrm{mL}$. No grupo de sobreviventes, a mediana obtida foi de $3 \mathrm{pg} / \mathrm{mL}$.

Chen T, et al. (2020) discorre que a insuficiência cardíaca e a lesão cardíaca aguda constituem condições comuns em se tratando de complicações da doença, sendo que dos 83 pacientes mortos pesquisados para insuficiência cardíaca, 41 apresentaram resultado positivo (49\%) e dos 94 pesquisados, também mortos, para dano cardíaco agudo, 72 evidenciaram resultado positivo (77\%). Isso ratifica o fato de que pacientes com comorbidades cardiovasculares pré-existentes caracterizam significativo número entre os mortos.

Os achados em torno do sistema cardiovascular, tanto em sua fisiopatologia quanto em suas manifestações, são, portanto, relevantes para uma melhor compreensão desta doença e de seus efeitos, assim como de possíveis protocolos de tratamento.

\section{CONSIDERAÇÕES FINAIS}

Diante da abordagem supracitada e do estudo discorrido, foi possível identificar a relação entre as comorbidades relacionadas ao sistema cardiovascular e a COVID-19. Verificou-se que os pacientes portadores de doenças cardíacas têm um prognóstico negativo diante da doença provocada pela infecção por SARS-CoV-2, em mais da metade dos casos ocorrendo o óbito. Observou-se que essa realidade é muito influenciada pelo uso de receptores da enzima conversora da angiotensina (ECA) pelo vírus para entrar na célula e que, mesmo prevendo que reduzir ou bloquear a expressão dessa enzima poderia ser uma forma de tratamento, não há evidências cientificas que embasem a suspensão de medicamentos com atividade na expressão da ECA. Além disso, estudos relatam casos de pacientes que desenvolveram lesões cardíacas secundárias pós-infecção por COVID-19. Todavia, as pesquisas ainda são insuficientes em tal âmbito se considerado o que foi descoberto e comprovado até o momento. Por fim, este estudo cumpre sua finalidade de observar e de relatar as características da infecção por SARS-CoV-2 em pacientes com comorbidades cardíacas, sejam previamente ou posteriormente à infecção viral. 


\section{REFERÊNCIAS}

1. BANSAL M. Cardiovascular disease and COVID-19. Diabetes \& Metabolic Syndrome: Clinical Research \& Reviews. 2020; 14(3): 247-250.

2. BARRASA H, et al. SARS-CoV-2 in Spanish Intensive Care Units: Early experience with 15-day survival in Vitoria. Anaesth Crit Care Pain Med. 2020; 39(5): 553-561.

3. BORGES GM, CRESPO CD. Aspectos demográficos e socioeconômicos dos adultos brasileiros e a COVID-19: uma análise dos grupos de risco a partir da Pesquisa Nacional de Saúde, 2013. Cad. Saúde Pública, 2020; 36(10): e00141020.

4. CHEN T, et al. Clinical characteristics of 113 deceased patients with coronavirus disease 2019: retrospective study. BMJ, 2020; m1091.

5. CHENG P, et al. Cardiovascular Risks in Patients with COVID-19: Potential Mechanisms and Areas of Uncertainty. CurrCardiol Rep, 2020; 22(5): 34.

6. CLERKIN KJ, et al. COVID-19 and Cardiovascular Disease. Circulation. 2020; 141(20): 1648-1655.

7. COSTA IBS da S, et al. O Coração e a COVID-19: O que o Cardiologista Precisa Saber. Arquivos Brasileiros de Cardiologia, 2020; 114(5): 805-16.

8. CRIVELARI NC, et al. Severe Cardiovascular Complications of COVID-19: a Challenge for the Physician. Int. J. Cardiovasc. Sci., 2020; 33(5): 572-581.

9. FERRARI F. COVID-19: Dados Atualizados e sua Relação Com o Sistema Cardiovascular. Arquivos Brasileiros de Cardiologia, 2020.

10. GORI T, et al. Perspective: cardiovascular disease and the Covid-19 pandemic. Basic Res Cardiol, 2020; 115(3):32.

11. GRANEMANN S. Crise econômica e a Covid-19: rebatimentos na vida (e morte) da classe trabalhadora brasileira. Trab. educ. Saúde, 2021; 19: e00305137

12. KAWAHARA LT, et al. Câncer e Doenças Cardiovasculares na Pandemia de COVID-19. Arq. Bras. Cardiol. 2020; 115(3): 547-557

13. PIVA S, et al. Clinical presentation and initial management critically ill patients with severe acute respiratory syndrome coronavirus 2 (SARS-CoV-2) infection in Brescia, Italy. Journal of Critical Care 58, 2020; 29-33

14. RENTE A, et al. Coronavírus e o Coração | Um Relato de Caso sobre a Evolução da COVID-19 Associado à Evolução Cardiológica. Arquivos Brasileiros de Cardiologia, 2020.

15. SOUTH AM, et al. COVID-19, ACE2, and the cardiovascular consequences. American Journal of Physiology-Heart and Circulatory Physiology, 2020; 318(5): H1084-90.

16. STRABELLI TMV, UIP DE. COVID-19 e o Coração: COVID-19 e o Coração. ABC Cardiol. 2020.

17. TAN W, ABOULHOSN J. The cardiovascular burden of coronavirus disease 2019 (COVID-19) with a focus on congenital heart disease. International Journal of Cardiology, 2020; 309: 70-7.

18. WANG D, et al. Clinical course and outcome of 107 patients infected with the novel coronavirus, SARS-CoV-2, discharged from two hospitals in Wuhan, China. CritCare, 2020; 24(1): 188.

19. ZHENG Y-Y, et al. COVID-19 and the cardiovascular system. Nat Rev Cardiol, 2020; 17(5): 259-60.

20. ZHOU F, et al. Clinical course and risk factors for mortality of adult in patients with COVID-19 in Wuhan, China: a retrospective cohort study. The Lancet, 2020; 395(10229): 1054-62. 